AperTO - Archivio Istituzionale Open Access dell'Università di Torino

\title{
Myths about nutrition in pregnancy
}

\section{This is the author's manuscript}

Original Citation:

\section{Availability:}

This version is available http://hdl.handle.net/2318/1561915

since 2017-05-15T09:54:05Z

Terms of use:

Open Access

Anyone can freely access the full text of works made available as "Open Access". Works made available under a Creative Commons license can be used according to the terms and conditions of said license. Use of all other works requires consent of the right holder (author or publisher) if not exempted from copyright protection by the applicable law. 
3 Alice Guggino ${ }^{1}$, Sara Barbero ${ }^{1}$,Valentina Ponzo $^{1}$, Elsa Viora ${ }^{2}$, Marilena Durazzo ${ }^{1}$, Simona Bo ${ }^{1}$

$4 \quad{ }^{1}$ Department of Medical Sciences, University of Turin, Italy;

$5 \quad$ 2Città della Salute e della Scienza S. Anna Hospital, Turin, Italy

6

$7 \quad$ Short running title: nutrition myths in pregnancy

8

9 Corresponding author: Simona Bo,

10 Department of Medical Sciences, University of Turin, Corso AM Dogliotti 14, 10126 Turin, Italy

11 Telephone +(39)(011)6336036 Fax+(39)(011)6635401 E-mail: simona.bo@unito.it

12

13 Keywords: dietician, education, myths, nutrition, pregnancy

14

Word count: text 750

16

17

18

19

20

21

22

23

24

25

26 
28 Many women have incorrect knowledge about nutrition in pregnancy owing to false beliefs derived

29 from popular practices. More than $90 \%$ of our cohort of pregnant women during early pregnancy

30 (<12 weeks of gestational age) gave at least one incorrect answer to the five questions relative to

31 common myths about nutrition in pregnancy. Education was inversely associated with the

32 percentage of incorrect answers, and the lowest percentage of any mistakes was found in the small

33 number of women who received nutritional information by a dietician.

34 In conclusion, the usual sources of information about nutrition in pregnancy are not adequate to overcome the false beliefs acquired by traditions. 


\section{Introduction}

Nutrition during pregnancy may influence the outcomes both of mother and foetus (Berti et al., 2010; Ricci E et al., 2010). Diet among pregnant women may be influenced by food preferences, ethnicity, age, education, income, parity, socio-cultural influences, household and community environment (Vieira Martins \& Almeida Remoaldo, 2007; Aubel, 2012). Many women hold wrong concepts about nutrition in pregnancy deriving from either false beliefs transmitted by parents or myths belonging to the popular tradition; despite this, few data relative to confined areas are available about this topic (Vieira Martins \& Almeida Remoaldo, 2007).

\section{Methods}

To investigate the prevalence and the characteristics of pregnant women believing in myths about diet in pregnancy, we interviewed all pregnant women consecutively admitted to the first trimester obstetric echography at the Ultrasound Unit of the Obstetrical Department of the S. Anna Hospital of Turin between January and July 2012. The procedures were in accordance to the Helsinki Declaration principles and the study protocol had been approved by the hospital committee. A semi-quantitative food-frequency questionnaire was administered to all women and data about age, parity, smoking habits, exercise and the source of information about diet in pregnancy were collected. Furthermore, four questions (which were all incorrect) and a fifth trap question (which was indeed correct) were added to the questionnaire as indicated below:

1)“Do you think that a glass of red wine should be advisable to improve blood circulation during pregnancy?”; 2)“Do you think that doubling food portions is necessary during pregnancy to satisfy energy requirements and ensure a healthy foetal growth?”; 3)“Do you consider appropriate assuming a sugar supplement (juices, candies..) in case of weakness or dizziness?”; 4)“Do you believe that herbal tea may be useful to avoid fluid retention?” and 5)“Do you think that consuming a medium sized portion of protein-rich foods (e.g. meat, fish, eggs, legumes..) twice a day is appropriate during the course of pregnancy?”. 
The association between incorrect answers and the other variables of interest was evaluated by a logistic regression model; $\mathrm{p}<0.05$ was considered statistically significant.

\section{Results}

Out of 526 women evaluated at our Hospital, 421 (80.0\%) accepted to participate (age $32.8 \pm 4.9$ years; gestational age 11.2 \pm 0.6 weeks); 171/421 (40.62\%) had graduated, $177 / 421$ (42.04\%) and 73/421 (17.34\%) had attended secondary and primary schools respectively. Most of them (65.8\%) reported sedentary habits ( $<2 \mathrm{~h} /$ week exercise), $12.3 \%$ were actual smoker and $8.8 \%$ regularly consumed a moderate amount of alcohol (15g/day).

Information about diet in pregnancy were obtained from multiple sources in $88.8 \%$ of cases, but $11.2 \%$ of women did not have any source of information. The former have provided multiple answers to this question: $23.8 \%$ from parents/friends, 35.9\% from TV/internet/newspapers, $69.4 \%$ from gynaecologist and 5.5\% from dietician.

Table I shows the prevalence of incorrect answers to the five questions: only $7.4 \%$ of the cohort correctly answered all questions. A significant inverse association between the graduation title and the percentage of incorrect answers was evident, even if the underestimation of protein requirements in pregnancy was common to all the education levels. The results did not change significantly after adjusting for age, although women in the lowest tertile of age (age $\leq 30$ years) showed a 2-fold higher risk of believing that doubling portions is correct during pregnancy $(\mathrm{OR}=2.29 ; 95 \% \mathrm{CI}$ 1.16-4.55, $\mathrm{p}=0.02)$

Finally, only obtaining information from dieticians was associated with a correct answer to the fifth question (OR=3.42; 95\%CI 1.41-8.28; $\mathrm{p}=0.007)$ independently of the educational level.

No significant association between parity, gestational age, alcohol intake, smoking habits, exercise level and incorrect answers was highlighted. 


\section{Discussion}

105 An unexpectedly high percentage of women (>90\%) reported at least one false belief about nutrition in pregnancy. Education was inversely associated with the percentage of incorrect answers; moreover the lowest percentage of mistakes $(<60 \%)$ was found in the small number (23/421) of women informed by dieticians. These results suggest that the usual sources of information about nutrition in pregnancy (gynaecologists included) are not adequate to overcome the false beliefs transmitted by popular practices and traditions.

We collected data from women during their first trimester of pregnancy and from a single obstetrical unit. Nevertheless, this unit is the biggest gynaecological centre in Turin and we cannot exclude that knowledge about nutrition could improve during the pregnancy. But it is a matter of fact that a healthy diet during the first months is critical to improve both maternal and foetal outcomes (Ramakrishnan et al., 2012).

These data, if confirmed by further studies, outline the need of appropriate nutritional education during early pregnancy, owing to their potential implications for the mothers as for the offspring.

\section{Declaration of Interest}

123 The authors declare that they have no conflicts of interest.

\section{Source of funding}

126 None 
132 Aubel J. 2012. The role and influence of grandmothers on child nutrition: culturally designated 133 advisors and caregivers. Maternal \& Child Nutrition 8: 19-35.

Berti C, Decsi T, Dykes F, Hermoso M, Koletzko B, Massari M, et al. 2010. Critical issues in setting micronutrient recommendations for pregnant women: an insight. Maternal \& Child Nutrition 2: 5-22.

Ramakrishnan U, Grant F, Goldenberf T, Zongrone A, Martorell R. 2012. Effect of women’s nutrition before and during early pregnancy on maternal and infant outcomes: a systematic review. Paediatric and Perinatal Epidemiology 26: S285-S301. small for gestational age birth: results from a retrospective case-control study in Italy. Maternal \& Child Nutrition 6: 297-305.

Vieira Martins F \& Almeida Remoaldo PC. 2007. Myths and beliefs during pregnancy in the Infirmiers 90: 75-85. 


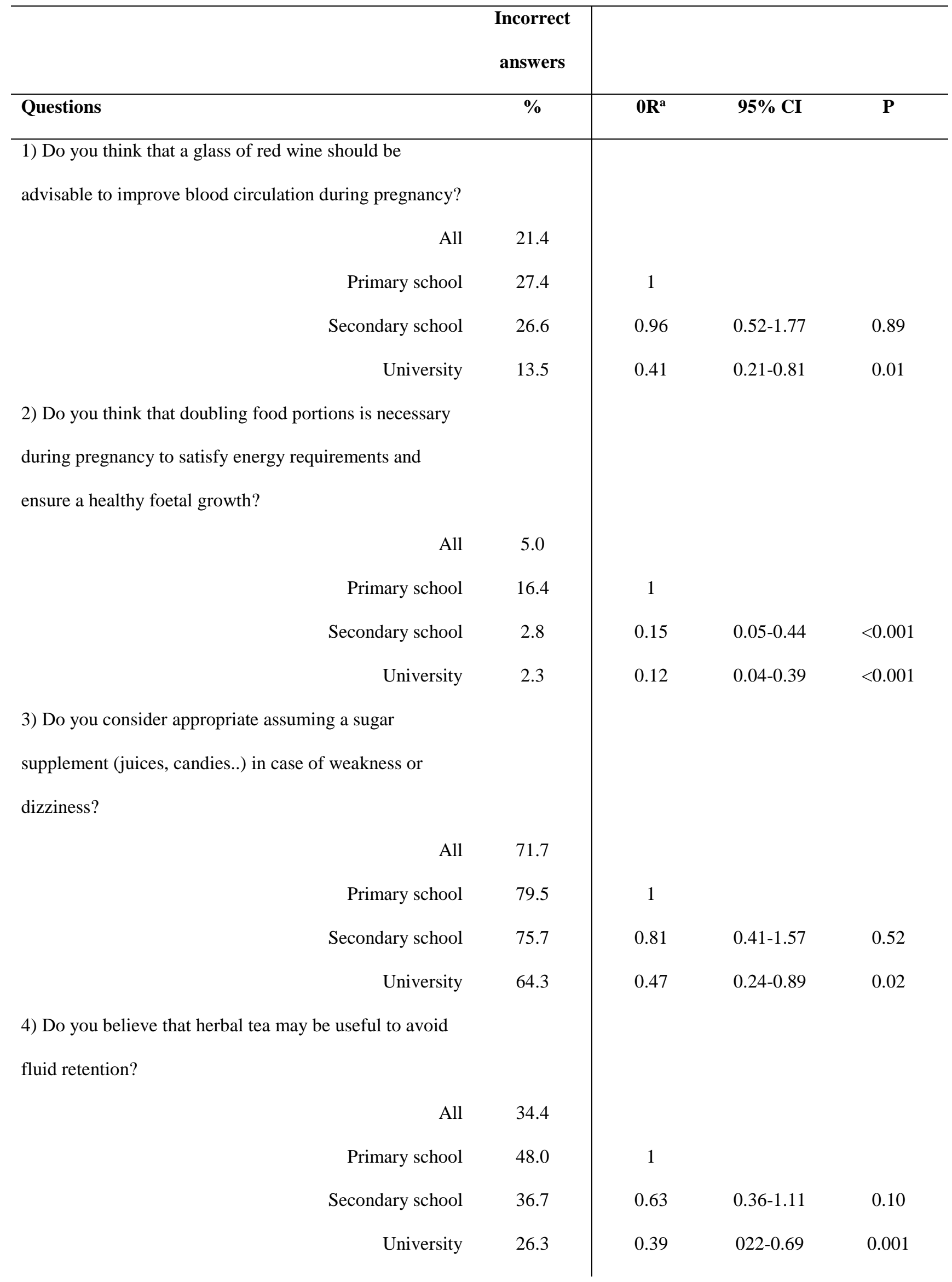


5) Do you think that consuming a medium sized portion of protein-rich foods (e.g. meat, fish, eggs, legumes..) twice a day is appropriate during the course of pregnancy?

All

62.9

Primary school

Secondary school

68.5

1

66.1

0.90

$0.50-1.61$

0.72

University

57.3

0.62

$0.34-1.10$

0.10

157

$158 \quad{ }^{a}$ OR evaluated by logistic regression analyses. 